\title{
Dispersion relation for a three-dimensional lamellar grating
}

\author{
J. T. Donohue \\ Centre d'Etudes Nucléaires de Bordeaux-Gradignan, Université Bordeaux 1, CNRS/IN2P3, BP 120, 33175 Gradignan, France \\ J. Gardelle \\ CEA, CESTA, F-33114 Le Barp, France \\ (Received 18 June 2010; revised manuscript received 6 May 2011; published 24 June 2011)
}

\begin{abstract}
A few years ago, Andrews and Brau (AB) presented the dispersion relation for a lamellar grating in two dimensions (2D) as a step in understanding coherent Smith-Purcell radiation. This involved solving Maxwell's equations both in the grooves and in the region above the grooves, where Floquet theory was used. The coupling with an electron beam was studied, and an expression for the gain as a function of current was derived. Their approach has been supported by $2 \mathrm{D}$ simulations using particle-in-cell (PIC) codes, and more recently by a demonstration experiment that used a wide grating. We present here the dispersion relation (in the absence of beam) of a grating in three dimensions, which turns out to be a relatively straightforward extension of the $\mathrm{AB}$ results. The predictions of this theory are compared with PIC simulations, and also with measurements of the transmission coefficient as a function of frequency. Extremely good agreement is observed.
\end{abstract}

DOI: 10.1103/PhysRevSTAB.14.060709

PACS numbers: 41.60.Cr, 52.65.Rr, 07.57.Hm

\section{INTRODUCTION}

In 1953 Smith and Purcell (SP) [1] sent an electron beam of about $300 \mathrm{keV}$ energy along the surface of a diffraction grating, and observed visible radiation which satisfied the condition they proposed

$$
\lambda=L(1 / \beta-\cos \theta) /|n|,
$$

where $\lambda$ denotes the wavelength of the radiation produced at angle $\theta$ with respect to the beam, the grating period is $L$, and $n$, the order of diffraction, is a negative integer (for SP radiation). The quantity $\beta=v / c$, where $v$ denotes the electron's velocity and $c$ the speed of light. Although many contributions to the understanding of this radiation have been made over the past half century, a renaissance of interest followed the publication of a theory for the production of interbunch coherent SP radiation by Andrews and Brau [2]. In this paper, the authors solved the Maxwell equations for a lamellar grating in 2D (the grooves were assumed infinitely long with no variation of the fields in their direction). They established the existence of an evanescent wave in the vicinity of the grating, and obtained the dispersion relation between frequency $\omega$ and axial wave number $k$ of this wave as the condition that a determinant vanishes. They showed that the intersection of the dispersion relation with the beam line, $\omega=v k$, occurs at a frequency $\omega$ less than the minimum allowed SP frequency. The interaction between beam and wave leads to beam

Published by the American Physical Society under the terms of the Creative Commons Attribution 3.0 License. Further distribution of this work must maintain attribution to the author(s) and the published article's title, journal citation, and DOI. bunching at that frequency, and the evanescent wave will be radiated from the ends of the grating. If the bunching is strong enough, harmonics of the bunching frequency may appear in the current, and this could produce coherent monochromatic radiation in a small interval around the corresponding SP angle. It was pointed out by $\mathrm{AB}$ that, for sufficiently low beam energy, the intersection would occur on that part of the dispersion relation where the slope $d \omega / d k$ is negative, as in a backward wave oscillator. The theory of Andrews and Brau was subsequently described in greater detail by them and their collaborators [3]. Support for their view was provided by simulations $[4,5]$ that used the 2D electromagnetic code MAGIC [6]. Shortly thereafter, Kumar and Kim [7] analyzed the problem using a different approach, but arrived at conclusions quite similar to those of AB. We should also mention the paper of Skrynnik et al. [8], who had performed a SP experiment at very low beam energies several years before, and found a dispersion relation that prefigured the results of $\mathrm{AB}$. It is also true that the analysis of the evanescent wave on a lamellar grating has long been available in the literature, notably in the treatise of Collin [9].

Despite the consensus concerning the $\mathrm{AB}$ theory, until a short time ago no experimental results supported their scenario. However, two recent experiments have reported evidence in its favor. The Vanderbilt-Vermont Photonics collaboration [10] observed the evanescent wave, but they used a grating equipped with sidewalls at the groove ends, which is not strictly 2D. At CEA-CESTA a demonstration experiment in the microwave domain without sidewalls has found results in agreement with the scenario of $A B$ [11]. In the latter the width of both the grating and the beam, $w$, was $10 \mathrm{~cm}$. Under these conditions the main results of 
3D MAGIC simulations were similar to those of the 2D simulations presented by us in Refs. [4,12]. We conclude that if $w \gg L$, the 2D theory should be approximately valid. In the recent experiment, $w=5 L$.

Several attempts have been made to study coherent SP radiation in three dimensions, since all gratings have finite width. Kesar [13] has rigorously calculated the effect of finite grating width on the SP radiation from an electron bunch, as compared to the infinite width limit. Kim and Kumar [14] extended their previous analysis to 3D and provided estimates of the beam parameters and minimum current for a setup similar to the Dartmouth terahertz experiments [15]. Dazhi Li and co-workers performed 3D simulations using MAGIC [16-18]. They showed that one could expect bunching for sufficiently large currents and that if sidewalls were placed at the ends of the grooves, the start current would be reduced by a factor of 2 . They noted that with sidewalls the transverse profile of the axial component of the electric field has a cosine form, vanishing at the sidewalls, so that it couples well to a narrow beam in the middle of the grating. Andrews, Jarvis, and Brau [19] (AJB) presented a 3D dispersion relation for a lamellar grating with sidewalls, assuming cosine profiles in the grooves, as found by Li et al. They examined in detail the dispersion relation for the grating used in the experiment described in Ref. [10], and showed that the predicted modes of operation for a beam of $30 \mathrm{keV}$ were quite different according to whether the 2D or their new 3D theory applied. Experiment favored the 3D prediction. Jarvis, Andrews, and Brau [20] have recently provided a fuller description of their theory. A key assumption in this theory is that the axial magnetic field is zero. Although their theory agrees with the experimental results reported in Ref. [10], we show in Sec. IIE that this hypothesis leads to a contradiction in the behavior of the component of the magnetic field normal to the grating plane, and the component of the electric field along the grooves. Both of these must vanish at the tops of teeth, at the bottoms of grooves, and at infinite distance above the grating. But we show that the AJB theory implies monotonic behavior, which is inconsistent with the required vanishing. Thus, the AJB theory cannot be correct, despite its success in interpreting the experimental results shown in Ref. [10].

Although these 3D effects are of recent interest in the SP community, they had been treated long ago by the microwave tube community. Microwave tubes such as the Orotron [21] and Ledatron [22] employ a grating in an open resonator. Gratings in waveguides are also widely used as filters, and also in so-called Cherenkov grating amplifiers. A 3D theory of a lamellar grating in a closed waveguide structure was given by McVey, Basten, Booske, Joe, and Scharer [23]. Considerable literature exists on this subject, and our results represent a small but significant modification of existing work. Indeed, by slightly changing our theory to include a flat roof and sidewalls, we recover the results of Ref. [23]. We then benchmarked our modified theory against their published results, and found extremely good agreement.

We present here our 3D dispersion relation for a mode that propagates with nonvanishing wave number $q$ in the direction of the grooves. We find that the most general solution, with an assumed $e^{i(q x-\omega t)}$ dependence, has zero electric field in the direction of the grooves, and that the dispersion relation is a simple generalization of the $2 \mathrm{D} \mathrm{AB}$ solution, in which for a given axial wave number $k$ the 3D frequency $\omega_{3 \mathrm{D}}(k, q)$ is given by

$$
\omega_{3 \mathrm{D}}(k, q)=\sqrt{\left[\omega_{2 \mathrm{D}}(k)\right]^{2}+(c q)^{2}},
$$

where $\omega_{2 \mathrm{D}}(k)$ is the $2 \mathrm{D} \mathrm{AB}$ frequency. In Ref. [14], Kim and Kumar anticipated this result, using their approach based on the reflection coefficient matrix for the grating. It is also embodied in the results of Ref. [23], but not in Refs. $[19,20]$. The case of sidewalls is then obtained by constraining the transverse wave number $q$ to take on discrete values and adding the contribution with $-q$, to produce standing waves of the form $\cos (q x), \sin (q x)$. In support of our theory we show the results of 3D MAGIC simulations as well as measurements of the transmission coefficient of the grating used in the experiment described in Ref. [11]. Extremely good agreement is found among theory, simulation, and measurements carried out at loops and nodes of the transverse waves.

In Sec. II we present our theory. Its predictions are compared in Sec. III, both with 3D MAGIC simulations and with measurements of the transmission coefficient of the grating. Our findings are summarized in the Conclusion.

\section{THREE-DIMENSIONAL LAMELLAR GRATING THEORY}

\section{A. Sommerfeld's approach}

In this section we follow the discussion of Sommerfeld in Sec. 20 of his classic textbook [24]. The subject is guided surface waves along a cylindrical surface of arbitrary section, and the results are explicit formulas for the transverse components in terms of the transverse derivatives of the longitudinal components $E_{x}$ and $H_{x}$. The $x$ axis (along the grooves) is chosen as the direction of propagation, and a form $e^{i(q x-\omega t)}$ is assumed for all components (we use $q$ rather than Sommerfeld's $h$ for the wave number and the convention real part). Although Sommerfeld uses generalized coordinates $u, v$ for the transverse directions, we need only Cartesian coordinates $y$ (normal to the plane of the grating), and $z$ (in the plane, perpendicular to the grooves), as shown in Fig. 1. In our notation, the results are (in vacuum, with $\varepsilon_{0} \mu_{0}=1 / c^{2}$ ) 


$$
\begin{aligned}
& E_{y}=i\left(q \frac{\partial E_{x}}{\partial y}+\mu_{0} \omega \frac{\partial H_{x}}{\partial z}\right) /\left(\varepsilon_{0} \mu_{0} \omega^{2}-q^{2}\right), \\
& E_{z}=i\left(q \frac{\partial E_{x}}{\partial z}-\mu_{0} \omega \frac{\partial H_{x}}{\partial y}\right) /\left(\varepsilon_{0} \mu_{0} \omega^{2}-q^{2}\right), \\
& H_{y}=i\left(q \frac{\partial H_{x}}{\partial y}-\varepsilon_{0} \omega \frac{\partial E_{x}}{\partial z}\right) /\left(\varepsilon_{0} \mu_{0} \omega^{2}-q^{2}\right), \\
& H_{z}=i\left(q \frac{\partial H_{x}}{\partial z}+\varepsilon_{0} \omega \frac{\partial E_{x}}{\partial y}\right) /\left(\varepsilon_{0} \mu_{0} \omega^{2}-q^{2}\right) .
\end{aligned}
$$

Here the longitudinal components are assumed to satisfy the wave equation,

$$
\left(\frac{\partial^{2}}{\partial y^{2}}+\frac{\partial^{2}}{\partial z^{2}}-q^{2}+\varepsilon_{0} \mu_{0} \omega^{2}\right) E_{x}(y, z)=0
$$

with an identical equation for $H_{x}$.

For the lamellar grating, we choose the plane $y=0$ as the top of the infinitely long and wide grating. Let $L, A$, and
$H$ denote the period, the groove width, and the groove depth, respectively. Our choice of axes differs from those used by $\mathrm{AB}$ in Ref. [2], and the correspondence is $x=$ $-z_{\mathrm{AB}}, y=y_{\mathrm{AB}}, z=x_{\mathrm{AB}}$. In a groove, $-H \leq y \leq 0,0 \leq$ $z \leq A$, the perfect conductor boundary conditions are satisfied by the following forms:

$$
\begin{aligned}
& H_{x}^{g}(y, z)=\sum_{n=0}^{\infty} H_{n}^{g} \cos \left(\frac{n \pi z}{A}\right) \frac{\cosh \left[\kappa_{n}(y+H)\right]}{\cosh \left(\kappa_{n} H\right)} \\
& E_{x}^{g}(y, z)=\sum_{n=1}^{\infty} E_{n}^{g} \sin \left(\frac{n \pi z}{A}\right) \frac{\sinh \left[\kappa_{n}(y+H)\right]}{\cosh \left(\kappa_{n} H\right)}
\end{aligned}
$$

where $\kappa_{n}^{2}=\left(\frac{n \pi}{A}\right)^{2}+q^{2}-\frac{\omega^{2}}{c^{2}}$, and the $H^{g}{ }_{n}$ and $E^{g}{ }_{n}$ are complex numbers to be determined. From Eq. (2.1), the remaining components may be written

$$
\begin{aligned}
& E_{y}^{g}(y, z)=i\left[\sum_{n=1}^{\infty}\left(q \kappa_{n} E_{n}^{g}-\mu_{0} \omega \frac{n \pi}{A} H_{n}^{g}\right) \sin \left(\frac{n \pi z}{A}\right) \frac{\cosh \left[\kappa_{n}(y+H)\right]}{\cosh \left(\kappa_{n} H\right)}\right] /\left(\frac{\omega^{2}}{c^{2}}-q^{2}\right), \\
& E_{z}^{g}(y, z)=i\left[\sum_{n=0}^{\infty}\left(q \frac{n \pi}{A} E_{n}^{g}-\mu_{0} \omega \kappa_{n} H_{n}^{g}\right) \cos \left(\frac{n \pi z}{A}\right) \frac{\sinh \left[\kappa_{n}(y+H)\right]}{\cosh \left(\kappa_{n} H\right)}\right] /\left(\frac{\omega^{2}}{c^{2}}-q^{2}\right), \\
& H_{y}^{g}(y, z)=i\left[\sum_{n=0}^{\infty}\left(q \kappa_{n} H_{n}^{g}-\varepsilon_{0} \omega \frac{n \pi}{A} E_{n}^{g}\right) \cos \left(\frac{n \pi z}{A}\right) \frac{\sinh \left[\kappa_{n}(y+H)\right]}{\cosh \left(\kappa_{n} H\right)}\right] /\left(\frac{\omega^{2}}{c^{2}}-q^{2}\right), \\
& H_{z}^{g}(y, z)=-i\left[\sum_{n=1}^{\infty}\left(q \frac{n \pi}{A} H_{n}^{g}-\varepsilon_{0} \omega \kappa_{n} E_{n}^{g}\right) \sin \left(\frac{n \pi z}{A}\right) \frac{\cosh \left[\kappa_{n}(y+H)\right]}{\cosh \left(\kappa_{n} H\right)}\right] /\left(\frac{\omega^{2}}{c^{2}}-q^{2}\right) .
\end{aligned}
$$

In the region above the grating, $0 \leq y$, Floquet forms are used for $H_{x}$ and $E_{x}$,

$$
\begin{aligned}
& H_{x}^{a}(y, z)=\sum_{p=-\infty}^{\infty} H_{p}^{a} e^{i(k+p K) z-\alpha_{p} y}, \\
& E_{x}^{a}(y, z)=\sum_{p=-\infty}^{\infty} E_{p}^{a} e^{i(k+p K) z-\alpha_{p} y},
\end{aligned}
$$

where $k$ denotes the axial wave number, $K=2 \pi / L, \alpha_{p}^{2}=(k+p K)^{2}+q^{2}-\frac{\omega^{2}}{c^{2}}$, and the $H_{p}^{a}, E_{p}^{a}$ are coefficients to be determined. Again the other components follow from Eq. (2.1):

$$
\begin{aligned}
& E_{y}^{a}(y, z)=\left[\sum_{p=-\infty}^{\infty}\left[-i q \alpha_{p} E_{p}^{a}-\mu_{0} \omega(k+p K) H_{p}^{a}\right] e^{-\alpha_{p} y+i(k+p K) z}\right] /\left(\frac{\omega^{2}}{c^{2}}-q^{2}\right), \\
& E_{z}^{a}(y, z)=\left[\sum_{p=-\infty}^{\infty}\left[-q(k+p K) E_{p}^{a}+i \mu_{0} \omega \alpha_{p} H_{p}^{a}\right] e^{-\alpha_{p} y+i(k+p K) z}\right] /\left(\frac{\omega^{2}}{c^{2}}-q^{2}\right), \\
& H_{y}^{a}(y, z)=\left[\sum_{p=-\infty}^{\infty}\left[-i q \alpha_{p} H_{p}^{a}+\varepsilon_{0} \omega(k+p K) E_{p}^{a}\right] e^{-\alpha_{p} y+i(k+p K) z}\right] /\left(\frac{\omega^{2}}{c^{2}}-q^{2}\right), \\
& H_{z}^{a}(y, z)=\left[\sum_{p=-\infty}^{\infty}\left[-q(k+p K) H_{p}^{a}-i \varepsilon_{0} \omega \alpha_{p} E_{p}^{a}\right] e^{-\alpha_{p} y+i(k+p K) z}\right] /\left(\frac{\omega^{2}}{c^{2}}-q^{2}\right) .
\end{aligned}
$$




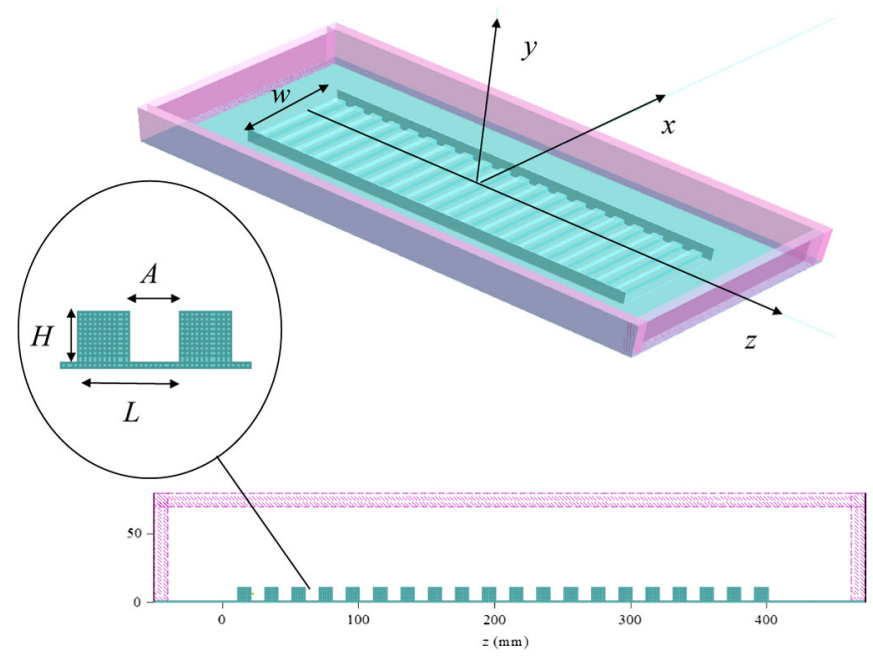

FIG. 1. Sketch of the 3D MAGIC simulation geometry, showing the grating in its surroundings, along with a transverse section indicating the grating parameters and the height of the simulation volume. The walls are composed of "free space," which absorbs radiation emerging from the grating.

Sommerfeld also points out that

$$
\begin{gathered}
\varepsilon_{0} \omega E_{z}+q H_{y}=-i \frac{\partial H_{x}}{\partial y}, \\
\mu_{0} \omega H_{z}-q E_{y}=i \frac{\partial E_{x}}{\partial y} .
\end{gathered}
$$

These equations facilitate the matching conditions at the top of the grating.

\section{B. Interface conditions and dispersion relation}

At the top of a tooth $(y \rightarrow 0+, A \leq z \leq L)$, the Floquet field components $E_{x}, E_{z}$, and $H_{y}$ must vanish for a perfect conductor, while at a groove $(y=0,0 \leq z \leq A)$ all Floquet components must be equal to the corresponding components in the groove. From Eqs. (2.8) and (2.9), we conclude that $E_{x}$ and $\frac{\partial H_{x}}{\partial y}$ vanish on top of a tooth and must be continuous across the groove, whereas $H_{x}$ and $\frac{\partial E_{x}}{\partial y}$ must match only at a groove. Multiplying the former by $e^{-i(k+p K) z} / L$ and integrating from 0 to $L$ yields two equations:

$$
\begin{gathered}
E_{p}^{a}=\frac{1}{L} \int_{0}^{A} d z e^{-i(k+p K) z} E_{x}^{g}(0, z) \\
=\frac{1}{L} \sum_{n=1}^{\infty} E_{n}^{g} J_{p n} \tanh \left(\kappa_{n} H\right), \\
H_{p}^{a}=\frac{-1}{\alpha_{p} L} \int_{0}^{A} d z e^{-i(k+p K) z} \frac{\partial H_{x}^{g}(0, z)}{\partial y} \\
=\frac{-1}{\alpha_{p} L} \sum_{n=0}^{\infty} H_{n}^{g} K_{p n} \kappa_{n} \tanh \left(\kappa_{n} H\right) .
\end{gathered}
$$

Here we have used the substitutions ( $K_{p n}$ was used by $\mathrm{AB}$ )

$$
\begin{aligned}
K_{p n} & =\int_{0}^{A} d z \cos \left(\frac{n \pi z}{A}\right) e^{-i(k+p K) z} \\
& =\frac{i(k+p K)\left[-1+(-1)^{n} e^{-i(k+p K) A}\right]}{\left[(k+p K)^{2}-\left(\frac{n \pi}{A}\right)^{2}\right]} \\
J_{p n} & =\int_{0}^{A} d z \sin \left(\frac{n \pi z}{A}\right) e^{-i(k+p K) z} \\
& =\frac{n \pi\left[-1+(-1)^{n} e^{-i(k+p K) A}\right]}{A\left[(k+p K)^{2}-\left(\frac{n \pi}{A}\right)^{2}\right]}
\end{aligned}
$$

Continuity of $H_{x}$ across the groove implies, upon multiplication by $\cos \left(\frac{n \pi z}{A}\right) / A$ followed by integration from $z=0$ to $A$,

$$
\begin{aligned}
H_{n}^{g} & =\frac{2}{\left(1+\delta_{0 n}\right) A} \int_{0}^{A} d z \cos \left(\frac{n \pi z}{A}\right) H_{x}^{g}(0, z) \\
& =\frac{2}{\left(1+\delta_{0 n}\right) A} \sum_{p=-\infty}^{\infty} H_{p}^{a} K_{p n}{ }^{*} .
\end{aligned}
$$

Multiplying $\frac{\partial E_{x}}{\partial y}$ by $\sin \left(\frac{n \pi z}{A}\right) / A$ followed by integration from $z=0$ to $A$, we find

$$
\begin{aligned}
E_{n}^{g} & =\frac{2}{\kappa_{n} A} \int_{0}^{A} d z \sin \left(\frac{n \pi z}{A}\right) \frac{\partial E_{x}^{g}(0, z)}{\partial y} \\
& =\frac{-2}{\kappa_{n} A} \sum_{p=-\infty}^{\infty} E_{p}^{a} \alpha_{p} J_{p n}{ }^{*} .
\end{aligned}
$$

Combining Eqs. (2.11) and (2.12), we write

$$
\begin{aligned}
H_{m}^{g}= & \frac{-2}{A L\left(1+\delta_{0 m}\right)} \sum_{n=0}^{\infty} H_{n}^{g} \kappa_{n} \tanh \left(\kappa_{n} H\right) \\
& \times\left(\sum_{p=-\infty}^{\infty} \frac{K_{p m}{ }^{*} K_{p n}}{\alpha_{p}}\right) .
\end{aligned}
$$

This expression may be rewritten as

$$
\sum_{n=0}^{\infty}\left(\bar{R}_{m n}-\delta_{m n}\right) H_{n}^{g}=0
$$

where the matrix elements $\bar{R}_{m n}$ are defined by

$$
\bar{R}_{m n}=\frac{-2 \kappa_{n} \tanh \left(\kappa_{n} H\right)}{A L\left(1+\delta_{0 m}\right)} \sum_{p=-\infty}^{\infty} \frac{K_{p m}{ }^{*} K_{p n}}{\alpha_{p}} .
$$

In order for a solution to exist, the matrix $\bar{R}$ must have an eigenvalue $=1$, and the solution for the $H_{n}^{g}$, to within multiplication by a complex constant, is the corresponding eigenvector. The matrix elements depend on the frequency $\omega$ and transverse wave number $q$ only through the combination $\omega^{2}-c^{2} q^{2}$, that appears in $\kappa_{n}$ and $\alpha_{p}$. This implies that if, for a given $k$, an eigenvalue of the matrix $=1$ when $q=0$ and $\omega=\omega_{2 \mathrm{D}}$, then the matrix has the same eigenvalue and eigenvector for arbitrary $q$ and 
$\omega=\sqrt{\omega_{2 \mathrm{D}}^{2}+c^{2} q^{2}}$. Our matrix $\bar{R}$ is related to the matrix $R$ of $\mathrm{AB}$ as follows:

$$
\bar{R}_{m n}=\kappa_{m}{ }^{-1} R_{m n} \kappa_{n},
$$

which implies that both have the same eigenvalues. The 3D problem for the coefficients $H_{n}^{g}$ is thus reduced to the 2D problem of $\mathrm{AB}$. The dispersion relation for arbitrary $q$ follows directly from that of $\mathrm{AB}$, following Eq. (1.2).

One may proceed in a similar way with Eqs. (2.10) and (2.13) to derive

$$
E_{m}^{g}=\frac{-2}{A L \kappa_{m}} \sum_{n=1}^{\infty} E_{n}^{g} \tanh \left(\kappa_{n} H\right)\left(\sum_{p=-\infty}^{\infty} \alpha_{p} J_{p m}{ }^{*} J_{p n}\right) .
$$

We may write this in matrix form, similar to Eq. (2.15),

$$
\sum_{n=1}^{\infty}\left(\bar{S}_{m n}-\delta_{m n}\right) E_{n}^{g}=0,
$$

where the matrix elements are (for $m, n>0$ )

$$
\bar{S}_{m n}=\frac{-2 \tanh \left(\kappa_{n} H\right)}{A L \kappa_{m}}\left(\sum_{p=-\infty}^{\infty} \alpha_{p} J_{p m}{ }^{*} J_{p n}\right) .
$$

If all the quantities $\alpha_{p}$ are real and positive (true if $\left.\omega_{2 D}<\operatorname{Min}[c k, c(K-k)], 0<k<K\right)$, the matrix $\bar{S}$ is negative definite, and has no eigenvalue $=1$. Furthermore, if some $\alpha_{p}$ are imaginary, the eigenvalues of $\bar{S}$ are no longer real, and thus there exists no mode with nonzero $E_{x}$. Thus, the only 3D modes for the grating are those with $E_{x}=0$, and these have $H_{z} \neq 0$.

Our result nonetheless represents a significant change compared to the $2 \mathrm{D}$ dispersion relation. In the latter, the grating acts as a low-pass filter, transmitting all frequencies less than the maximum, which occurs at the symmetry point, $k=K / 2$. In contrast, the minimum frequency in 3D is $c q$. The system thus acts as a bandpass filter, with a lower cutoff $c q$, and an upper cutoff $\sqrt{\left[\omega_{2 \mathrm{D}}(K / 2)\right]^{2}+c^{2} q^{2}}$. It follows also that the 3D group velocity in the $z$ direction is given by

$$
v_{g 3 \mathrm{D}}=v_{g 2 \mathrm{D}} \frac{\omega_{2 \mathrm{D}}}{\omega_{3 \mathrm{D}}} .
$$

\section{Transverse standing waves and sidewalls}

It should also be noted that a second solution with the same $\omega, k$ exists, but where $q \rightarrow-q$. By superposition, one may form the symmetric and antisymmetric combinations, which correspond to standing waves in the grooves. We also introduce the magnetic induction $\vec{B}=\mu_{0} \vec{H}$ to facilitate comparison with our simulations. All five nonzero components may be written in terms of $B_{x}$ and its derivatives.
Symmetric modes:

$$
\begin{aligned}
\vec{E}(\vec{x}, t)= & \frac{i \omega}{\varepsilon_{0} \mu_{0} \omega^{2}-q^{2}}\left(\frac{\partial B_{x}(y, z)}{\partial z} \hat{e}_{y}-\frac{\partial B_{x}(y, z)}{\partial y} \hat{e}_{z}\right) \\
& \times \cos (q x) e^{-i \omega t}, \\
\vec{B}(\vec{x}, t)= & \left(\frac{-q \sin (q x)}{\varepsilon_{0} \mu_{0} \omega^{2}-q^{2}}\left(\frac{\partial B_{x}(y, z)}{\partial y} \hat{e}_{y}+\frac{\partial B_{x}(y, z)}{\partial z} \hat{e}_{z}\right)\right. \\
& \left.+B_{x}(y, z) \cos (q x) \hat{e}_{x}\right) e^{-i \omega t} .
\end{aligned}
$$

From these one may derive the relations among components,

$$
\begin{aligned}
& B_{z}(\vec{x}, t)=\frac{q \tan (q x)}{\omega} E_{y}(\vec{x}, t-T / 4), \\
& B_{y}(\vec{x}, t)=\frac{q \tan (q x)}{\omega} E_{z}(\vec{x}, t+T / 4),
\end{aligned}
$$

where the period $T$ is $2 \pi / \omega$.

Antisymmetric modes:

$$
\begin{aligned}
\vec{E}(\vec{x}, t)= & \frac{i \omega}{\varepsilon_{0} \mu_{0} \omega^{2}-q^{2}}\left(\frac{\partial B_{x}(y, z)}{\partial z} \hat{e}_{y}-\frac{\partial B_{x}(y, z)}{\partial y} \hat{e}_{z}\right) \\
& \times \sin (q x) e^{-i \omega t}, \\
\vec{B}(\vec{x}, t)= & {\left[\frac{q \cos (q x)}{\varepsilon_{0} \mu_{0} \omega^{2}-q^{2}}\left(\frac{\partial B_{x}(y, z)}{\partial y} \hat{e}_{y}+\frac{\partial B_{x}(y, z)}{\partial z} \hat{e}_{z}\right)\right.} \\
& \left.+B_{x}(y, z) \sin (q x) \hat{e}_{x}\right] e^{-i \omega t} .
\end{aligned}
$$

These imply

$$
\begin{aligned}
& B_{z}(\vec{x}, t)=\frac{q}{\omega \tan (q x)} E_{y}(\vec{x}, t+T / 4), \\
& B_{y}(\vec{x}, t)=\frac{q}{\omega \tan (q x)} E_{z}(\vec{x}, t-T / 4) .
\end{aligned}
$$

If the grating, instead of being infinitely wide, were bounded by perfectly conducting walls at $x= \pm w / 2$, the vanishing at the walls of $B_{x}$ would impose that symmetric modes

$$
\begin{aligned}
q & =(2 m+1) \pi / w, \quad m=0,1,2, \ldots, \\
f_{3 \mathrm{D}}(k) & =\sqrt{\left[f_{2 \mathrm{D}}(k)\right]^{2}+\left(\frac{\left(m+\frac{1}{2}\right) c}{w}\right)^{2},}
\end{aligned}
$$

antisymmetric modes:

$$
\begin{aligned}
q & =2 n \pi / w, \quad n=1,2, \ldots, \\
f_{3 \mathrm{D}}(k) & =\sqrt{\left[f_{2 \mathrm{D}}(k)\right]^{2}+\left(\frac{n c}{w}\right)^{2}} .
\end{aligned}
$$

In Fig. 2 we display the dispersion relation for our grating, with $L=2 \mathrm{~cm}, A=H=1 \mathrm{~cm}$, and width $w=$ $10 \mathrm{~cm}$, if it had sidewalls. The 2D relation is shown along with the first four symmetric modes $m=0,1,2,3$ and the 


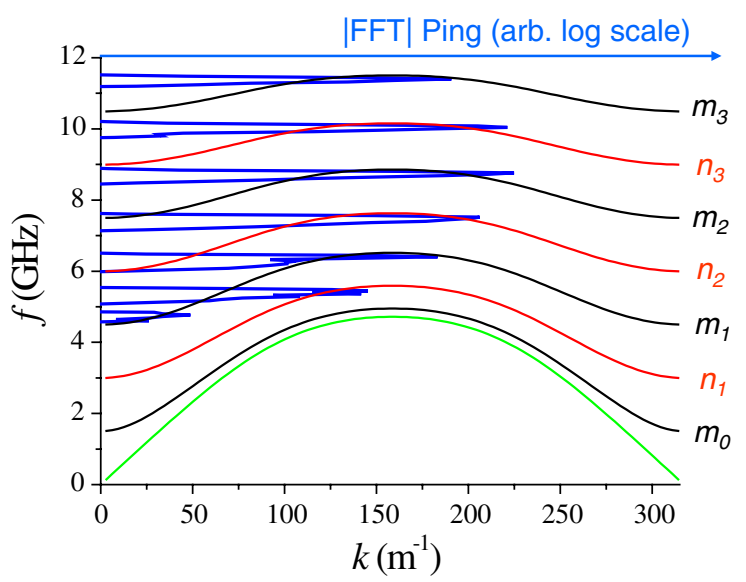

FIG. 2. The 2D dispersion relation for the grating is shown in green. For the grating with sidewalls, the 3D symmetric higher modes are shown in black, and the antisymmetric modes in red. The blue curve shows the FFT of a ping, which correlates very well with the maxima of these seven branches of the dispersion relation.

antisymmetric modes $n=1,2,3$. Also shown are the results of "pings," which are described in the Sec. III.

\section{Computations of fields}

This theory makes many predictions that are readily tested with the help of numerical codes that satisfy the Maxwell equations, such as the particle-in-cell (PIC) code MAGIC. In addition, since the grating used in Ref. [11] is well suited to the microwave frequency range, measurements of the transmission coefficient between any two points just above the top of the grating may be performed using a standard network analyzer. Typically the network analyzer measures the transmission coefficient as a function of frequency, for frequencies up to $10 \mathrm{GHz}$. Provided we can calculate the electromagnetic field components (to within an unknown complex constant), we can compare the theory with simulations.

Although we have concentrated on the dispersion relation, our theory actually can be used to compute the function $B_{g}(y, z)$ which determines the magnetic field component $B_{x}$ in the groove. With the help of the program MATHEMATICA [25], we compute a $7 \times 7$ approximation to the matrix $\bar{R}$, and for fixed $k$, seek the value of $\omega$ such that the determinant of $\bar{R}-\Im$ vanishes. The eigenvector of eigenvalue zero then provides the quantities $B_{n}^{g}$, for $n=0$ to 6 . These are normalized such that $\sum_{n=0}^{6}\left|B_{n}^{g}\right|^{2}=$ 1 , and the phase can be chosen such that $B_{0}^{g}$ is real. For our grating, $0.998<B_{0}^{g}<1$ for all wave numbers, which means that the $n=0$ term dominates the sum. For the electric fields in the groove this dominance is less pronounced, since the relevant coefficients for $E_{y}$ are $n B_{n}^{g}$ and for $E_{z}$ are $\kappa_{n} B_{n}^{g}$. The resulting series converge less rapidly. Consequently, our calculation of the function $B_{x}$ in the groove is accurate, that of $E_{z}$ somewhat less accurate, while that of $E_{y}$ is still less so, since the dominant $n=0$ term is absent. We test the internal consistency of our approach by computing the Floquet components, $B_{p}^{a},-10<p<10$, and from these the fields above the grating. Comparing the fields in the groove with those above at the interface $y=0$, we observe that the agreement for $B_{x}$ is good, that for $E_{z}$ acceptable, while the rather spiky behavior of $E_{y}$ is only approximately continuous. While taking more terms in the approximation would help things, we have confidence in our ability to calculate the fields with reasonable, if not perfect, accuracy.

\section{E. Comment on the AJB theory}

As we stated in the Introduction, in Ref. [19] AJB proposed a 3D theory for a grating with sidewalls that leads to a different dispersion relation. In particular, it does not satisfy our Eq. (1.2). The authors assumed that the axial component of the magnetic field vanishes, i.e., $B_{z}=0$. This is a valid hypothesis in $2 \mathrm{D}$, and AJB assumed it would also hold in 3D. Our solution, which we claim is of general validity, does not allow this. However, a more direct proof is required, and we outline it here. We assume that the magnetic field of $\mathrm{ABJ}$ may be written, for a sidewall grating, and omitting the $e^{-i \omega t}$ factor, as

$\vec{B}(x, y, z)=\cos (q x) b_{x}(y, z) \hat{e}_{x}+\sin (q x) b_{y}(y, z) \hat{e}_{y}$.

The vanishing of the divergence implies

$$
\frac{\partial b_{y}(y, z)}{\partial y}=q b_{x}(y, z) .
$$

Computations show that the function $b_{x}(y, z)$ has a fixed sign as a function of $y$. In particular, it has a maximum on top of a tooth, and then decays rapidly with increasing $y$, showing no oscillatory behavior. It follows from Eq. (2.31) that the derivative of $b_{y}(y, z)$ with respect to $y$ is also of fixed sign, meaning that $b_{y}(y, z)$ is monotonically increasing or decreasing. But the boundary conditions require $b_{y}(y, z)$ to vanish on top of a tooth and at the bottom of a groove, while the evanescent behavior at infinite $y$ requires it to vanish there. Clearly, this is impossible for a monotonic function, and a contradiction is obtained. Similar arguments can be made to show that the component $E_{x}$, which must also vanish on top of all teeth, at the bottom of all grooves, and as $y \rightarrow \infty$ is a monotonic function of $y$. We conclude that the basic hypothesis assumed by AJB leads to contradictory behavior for both $B_{y}$ and $E_{x}$.

\section{COMPARISON WITH SIMULATIONS AND MEASUREMENTS}

In support of our theory, we present some results obtained with simulations, together with transmission measurements performed on the grating using a network analyzer. In the 3D MAGIC simulations, the grating 
was chosen to have the parameters of that used in the demonstration experiment, i.e., twenty periods of length $L=2 \mathrm{~cm}, A=H=1 \mathrm{~cm}$, and width $w$ of the grating = $10 \mathrm{~cm}$. Its surface was assumed to be a perfect conductor, with vanishing tangential electric field and normal magnetic field components. In the simulations, MAGIC calculated all six components of the electromagnetic field. In order to get a rough idea of the transmission properties, a ping or short burst of localized electromagnetic field was excited in an upstream groove. The field then propagated down the grating, and time signals of the magnetic field component $B_{x}$ were observed in a downstream groove. From the fast Fourier transform (FFT) of this time signal, the spectrum of frequencies that can propagate is obtained at once. Then, having seen which frequencies are likely to propagate well, we simulate a monochromatic current source at a given frequency inserted in the first groove, and use the various MAGIC tools to study the properties of the fields that propagate downstream. Among these are the time histories of the fields at a point, the space dependence of a component along a given line in space (parallel to one of the axes) at fixed time, and contour maps of any plane of the grating (whose normal lies along an axis) for any field component at a fixed time. With the FFT capacity of MAGIC the frequency and wave number dependence of the signals may be determined, in order to test the theoretical predictions of the previous section.

A network analyzer model HP 8510 was used to measure the transmission of the grating surface wave between two arbitrary points, typically chosen to be just above a groove, at small values of the coordinate $y$. Most such measurements were performed with sidewalls, realized by fastening conducting planes at the ends of the grooves. This is indicated in Fig. 3, which shows a photograph of the setup. As shown, the connections were terminated by short linear antennas, which were oriented to excite and receive the component $E_{z}$. In addition, some simulations were performed with open-ended grooves, since that was the configuration used in the experiment.

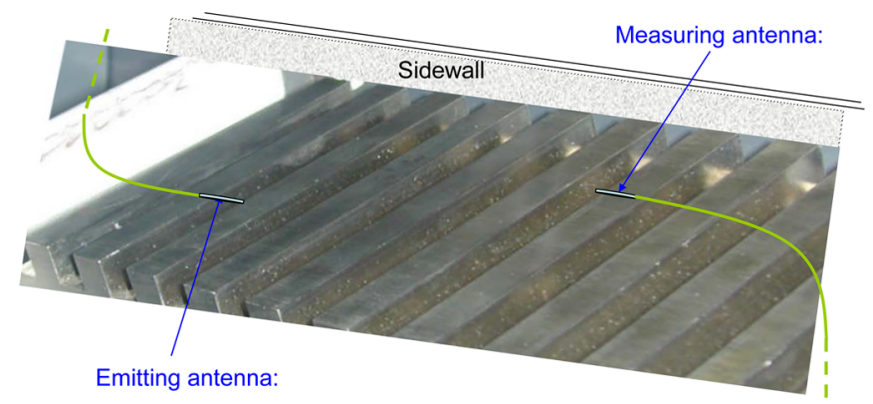

FIG. 3. Photograph of the grating during measurements of the transmission coefficient, showing the antennas connected to the network analyzer. One sidewall is drawn to show its position.

\section{A. Response of grating to a ping in simulations}

If a very short burst of current (ping) is used to excite the surface waves of the grating, the corresponding frequency spectrum will be broad. However, only those frequencies that satisfy the dispersion relation can propagate down the grating. By observing the field $B_{x}$ at a point downstream from the current source, and performing an FFT, one sees immediately what bands of frequencies are allowed. In 2D simulations, all frequencies up to the maximum (approximately $4.7 \mathrm{GHz}$ for our grating) are observed, but none above this. In contrast, the signals observed in 3D simulations show a series of allowed frequency bands, extending well beyond the 2D limit. When the grating has sidewalls, well-defined transverse modes with nodes and loops are seen. In Fig. 4(a) are shown the transverse profiles of $B_{x}$ for the four lowest symmetric modes, with $2 m$ internal nodes; the three lowest antisymmetric modes with $2 n-1$ internal nodes are shown in 4(b). The nodes and loops are indicated by solid circles and triangles, respectively. If either the current source is placed at a node or the observation point occurs at a node, the corresponding mode will not be excited (or only weakly, if the positioning is imperfect). Conversely, if both are placed at loops of a mode, the signal
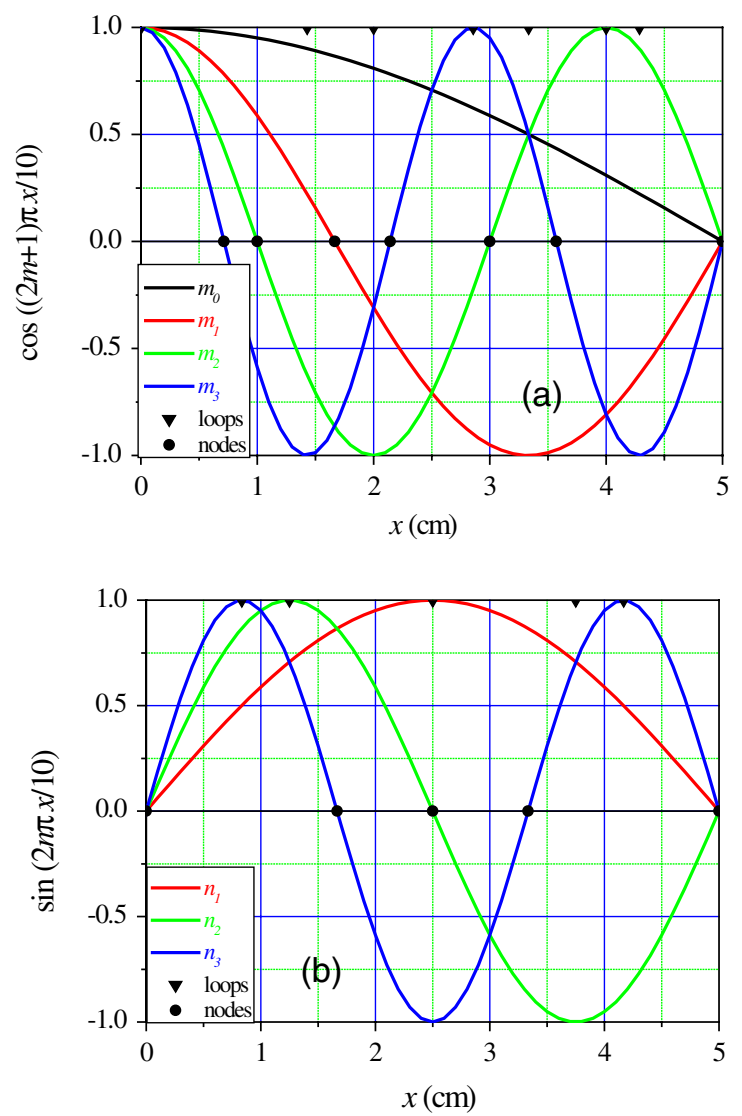

FIG. 4. Transverse profiles of the four lowest symmetric modes for the grating with sidewalls (a), and the three lowest antisymmetric modes (b). The nodes and loops are indicated. 
should be prominent. To illustrate this, we show in Fig. 5 for low frequencies and Fig. 6 for high frequencies the spectra corresponding to 11 different pings. The left-hand sides of these figures show the modulus of the FFT for the pings, while the right-hand sides show the modulus of the measured transmission coefficients. We used six distinct transverse positions for both emission and observation:
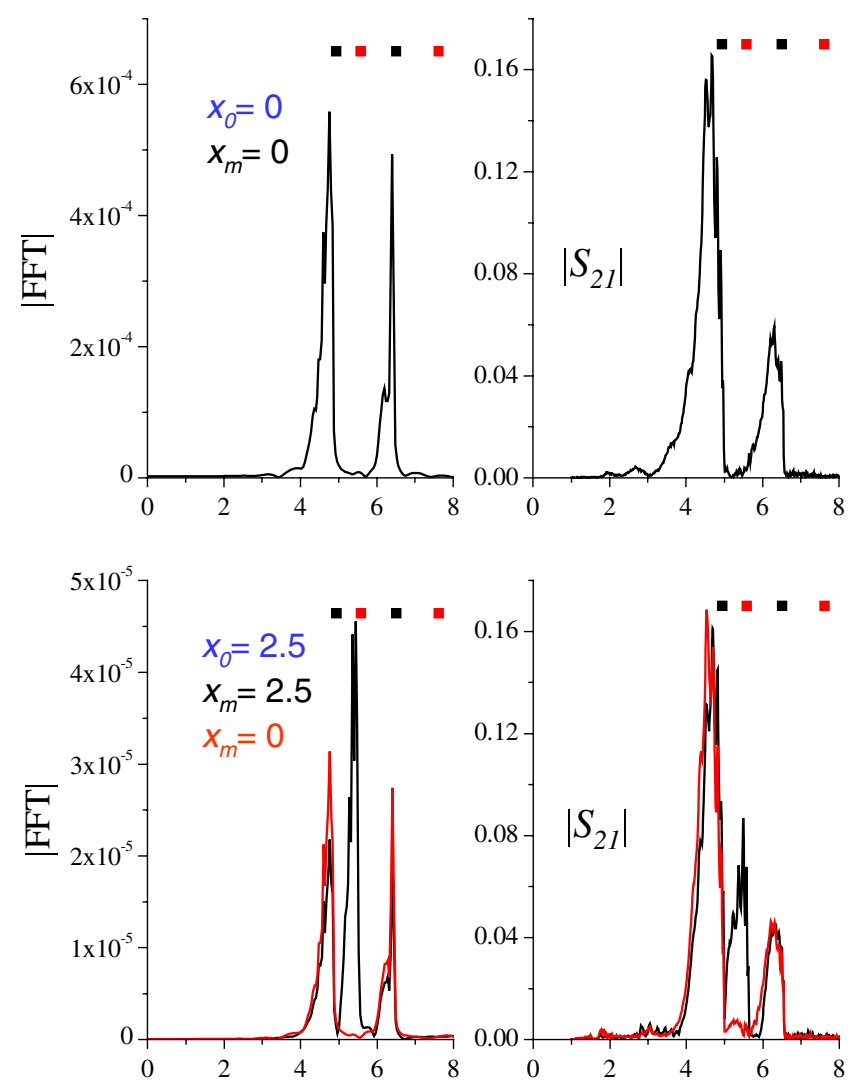

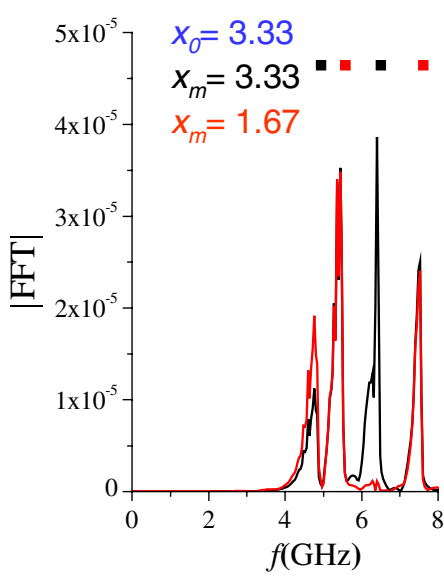

(a)

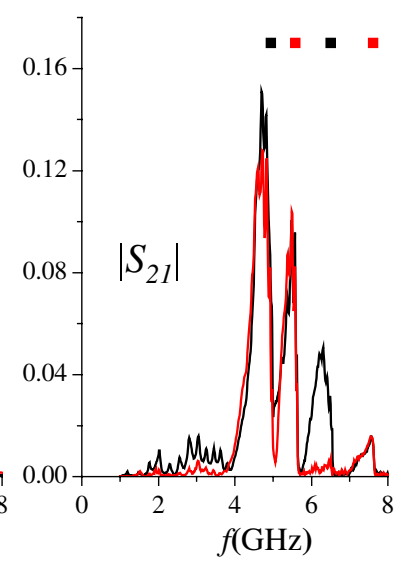

(b)
FIG. 5. (a) The moduli of the FFTs of pings emitted at transverse position $x_{0}$, and observed at $x_{m}$. (b) The moduli of the measured transmission coefficient $S_{21}$ for the same emission and measurement points. The theoretical band heads for symmetric (black) and antisymmetric modes (red) are indicated by small squares. See also Table I.

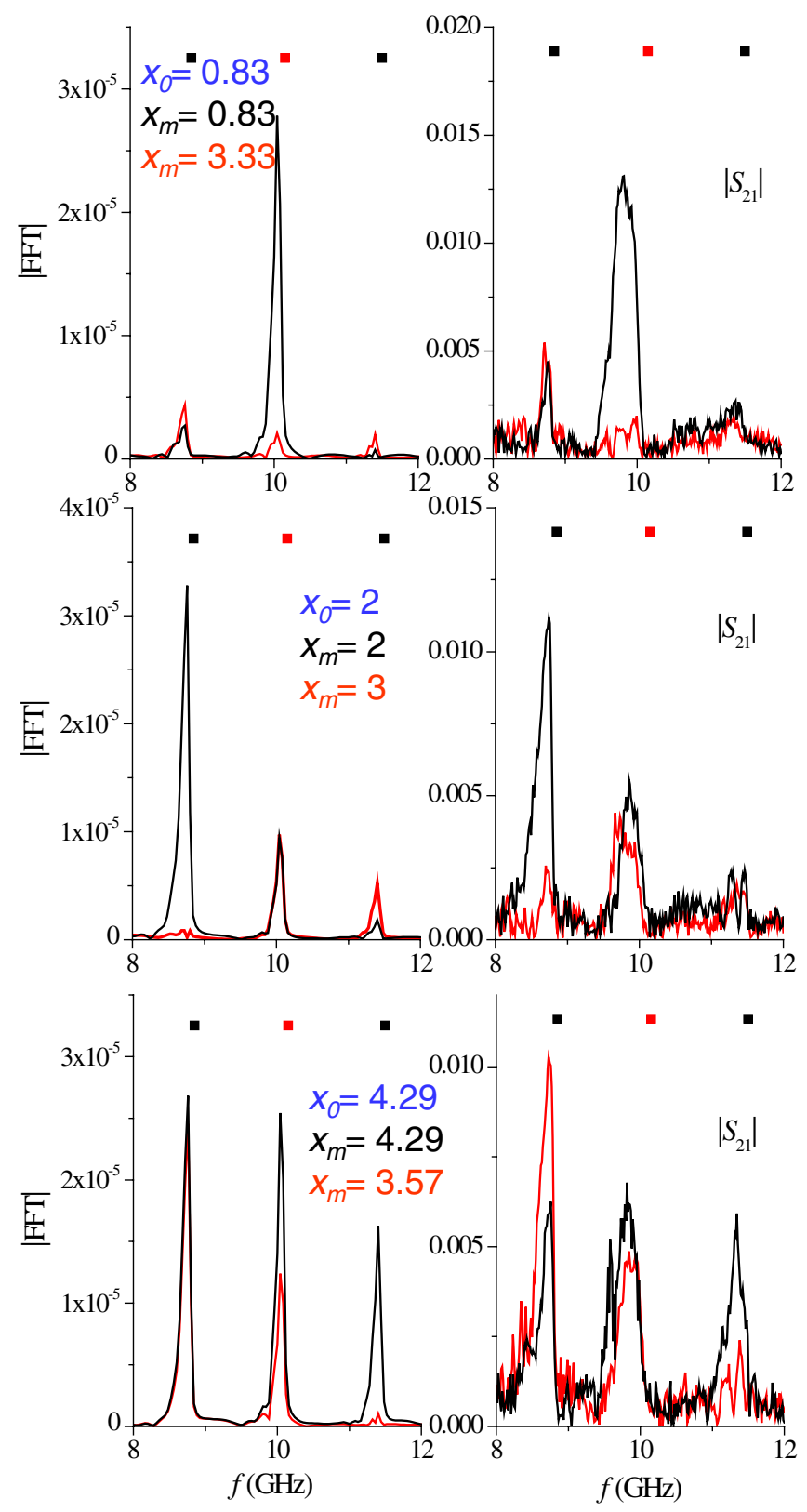

(a)

(b)

FIG. 6. Same as Fig. 5, except for different emission and measurement points, and a higher frequency range.

$0,0.83,2,2.5,3.33$, and $4.29 \mathrm{~cm}$. There were five additional emission-observation pairs: $(0.83,3.33),(2,3),(2.5,0)$, $(3.33,1.67)$, and $(4.29,3.57)$. In no case where the theory predicts zero signal is any seen, and at several places where loops are present at both emission and measurements, above average signals are seen. We have shown in Fig. 2 that the band heads occur at values corresponding to the formula $\sqrt{\left[f_{2 \mathrm{D}}(K / 2)\right]^{2}+\left[\left(m+\frac{1}{2}\right) c / w\right]^{2}}, \quad m=0,1,2,3$, for the symmetric modes, and $\sqrt{\left[f_{2 \mathrm{D}}(K / 2)\right]^{2}+(n c / w)^{2}}$ for the antisymmetric modes, with $n=1,2,3$. The network analyzer measurements are generally quite consistent 
TABLE I. Nodes and loops of grating with sidewalls.

\begin{tabular}{lllllll}
\hline \hline$x(\mathrm{~cm})$ & $x / w$ & Cosine node & Cosine loop & Sine node & Sine loop & Nearby \\
\hline 0 & 0 & & All $m$ & All $n$ & & \\
0.83 & $1 / 12$ & & & $n=3$ & Nodes $m=2,3$ \\
1.67 & $1 / 6$ & $m=1$ & & & & Node $m=3$ \\
2 & $1 / 5$ & & $m=2$ & & & \\
2.5 & $1 / 4$ & & & $n=2$ & & \\
3 & $3 / 10$ & $m=2$ & & & & \\
3.33 & $1 / 3$ & & $m=1$ & $n=3$ & & Loop $n=2$ \\
3.57 & $5 / 14$ & $m=3$ & & & & Loop $n=3$ \\
4.29 & $3 / 7$ & & $m=3$ & & & \\
\hline \hline
\end{tabular}

with the simulations, even though they are not strictly comparable. The measurements provide experimental support for both our theory and our simulations.

For the determined reader, we indicate in Table I the loops and nodes that are expected on the basis of our theory. In addition, some emission points $\left(x_{0}\right)$ and measurement points $\left(x_{m}\right)$ are quite close to either nodes or loops, and these are also indicated. All predictions may be verified in the Figs. 5 and 6.

When the grating has sidewalls, the transverse modes are imposed by the condition that $B_{x}$ vanishes there. However, in the experiment of Ref. [11], the grating had no sidewalls, and it is of interest to ask how the fields vary in the transverse direction. We have performed simulations with no sidewalls, and a selection of our results is shown in
Fig. 7. To our surprise, the ping results are quite similar to those in the sidewall case, with clearly visible band heads. The cutoff frequencies are different, of course, with the lowest band head around $4.74 \mathrm{GHz}$. This only marginally exceeds the $4.71 \mathrm{GHz} 2 \mathrm{D}$ cutoff, and it suggests a $\cos (q x)$ behavior with a small value of $q$, of order $0.1 \mathrm{~cm}^{-1}$. If the exciting antenna is placed at the middle of the grating, only symmetric modes are generated, with band heads at 4.74, 5.5, and 7.25 GHz. With asymmetric excitation, two additional antisymmetric bands are found, with maximum frequencies of 4.9 and $6.3 \mathrm{GHz}$. Although we do not understand why these results occur without sidewalls, we make the empirical observation that, if one imposes that $B_{x}$ have a loop at $x=5.5 \mathrm{~cm}(5 \mathrm{~mm}$ beyond the end of the groove), then the transverse wave numbers $q_{n}$ would be
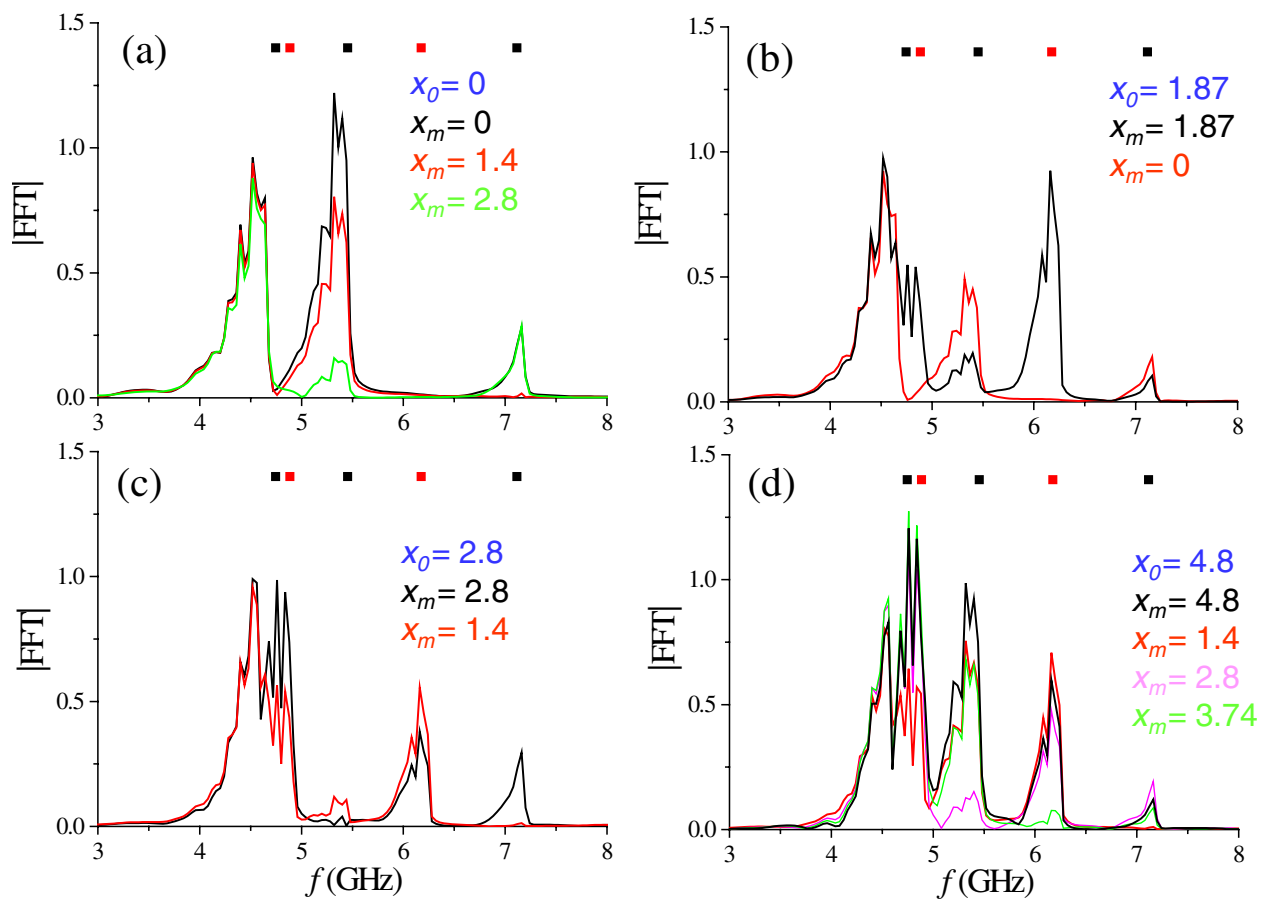

FIG. 7. The moduli of FFTs of pings emitted at transverse position $x_{0}$, and observed at $x_{m}$ for the grating without sidewalls. Bands with sharp cutoffs are clearly visible, just as for the grating with sidewalls. The empirical values for the band heads are again indicated for symmetric and antisymmetric modes. See also Table II. (a) $x_{0}=0$ (symmetric modes only), (b) $x_{0}=1.87$, (c) $x_{0}=2.8$, and (d) $x_{0}=4.8$. 
TABLE II. Nodes and loops of grating without sidewalls.

\begin{tabular}{llllll}
\hline \hline$x(\mathrm{~cm})$ & Cosine node & Cosine loop & Sine node & Sine loop & Nearby \\
\hline 0 & & $n=0,2,4$ & $n=1,3$ & & \\
1.4 & $n=4$ & & $n=3$ & Loop $n=3$ \\
1.87 & & $n=4$ & & \\
2.8 & $n=2$ & $n=3$ & & Loop all $n>0$ \\
3.7 & & & & \\
4.8 & & & & & \\
\hline \hline
\end{tabular}

$n \pi / 11 \mathrm{~cm}^{-1}, n=1,2,3$, and 4 . The corresponding band heads and transverse modes would be $\left[4.91 \mathrm{GHz}, \sin \left(q_{1} x\right)\right]$, [5.46 GHz, $\left.\cos \left(q_{2} x\right)\right],\left[6.27 \mathrm{GHz}, \sin \left(q_{3} x\right)\right]$, and [7.25 GHz, $\left.\cos \left(q_{4} x\right)\right]$. This is close to what we observe. In Rayleigh's "Theory of Sound" Sec. 312 [26], one learns that the note of a cylindrical organ pipe of radius $R$ behaves as if there were a loop at the open end, and the length were increased by $8 R / 3 \pi$. If the analogy with acoustics were valid, our results could be interpreted as a similar phenomenon.

We indicate in Table II those values of $n$ for which nodes or loops are expected to occur at points of emission or measurement, if the transverse wave numbers suggested above were correct. The agreement between these predictions and the simulations is reasonable, which supports our conjecture for the transverse profiles. Nevertheless, we cannot justify our empirical values of the transverse wave numbers $q_{n}$ from first principles.

\section{B. Results with monochromatic current driver}

Although the use of pings permits us to test the response of the grating over a broad range of frequency, a more sophisticated method of investigation is to insert a monochromatic current driver at some point in a groove, and thereby excite a simulated evanescent wave of definite frequency. If the source is placed at the midpoint of a groove, then only symmetric transverse modes will be excited. As may be seen from Fig. 2, the dispersion relation is such that if $k$ is an allowed wave number for a given frequency, then so is $K-k$. Thus, both forward and backward evanescent waves are generated, with unknown relative amplitudes. Reflections at both ends of the gratings also occur, again leading to a mixture of forward and backward waves. Various diagnostic tools available in MAGIC may then be used to examine details of the simulation, with the aim of testing our theory. For example, we show in Fig. 8 the results of four 3D simulations of our grating with sidewalls, with symmetric excitation at frequencies $4.75,6.4,8.7$, and $11.3 \mathrm{GHz}$. The current driver was placed in the first groove on the left, at $x=0$. Instantaneous contour maps of the field $B_{x}$ in the $x-z$ plane are shown, with $y=-2 \mathrm{~mm}$. On the right of the figure are shown the four lowest symmetric mode branches of dispersion relation for the grating. The standing wave transverse modes are clearly visible, with $0,2,4$, and 6 internal nodes, depending on the frequency. The positions of these modes on the different branches of the 3D dispersion relation are indicated by arrows. Although the wave numbers were determined from the FFT of the spatial dependence (not shown), crude estimations can be made from the contour plots by counting periods between recurring

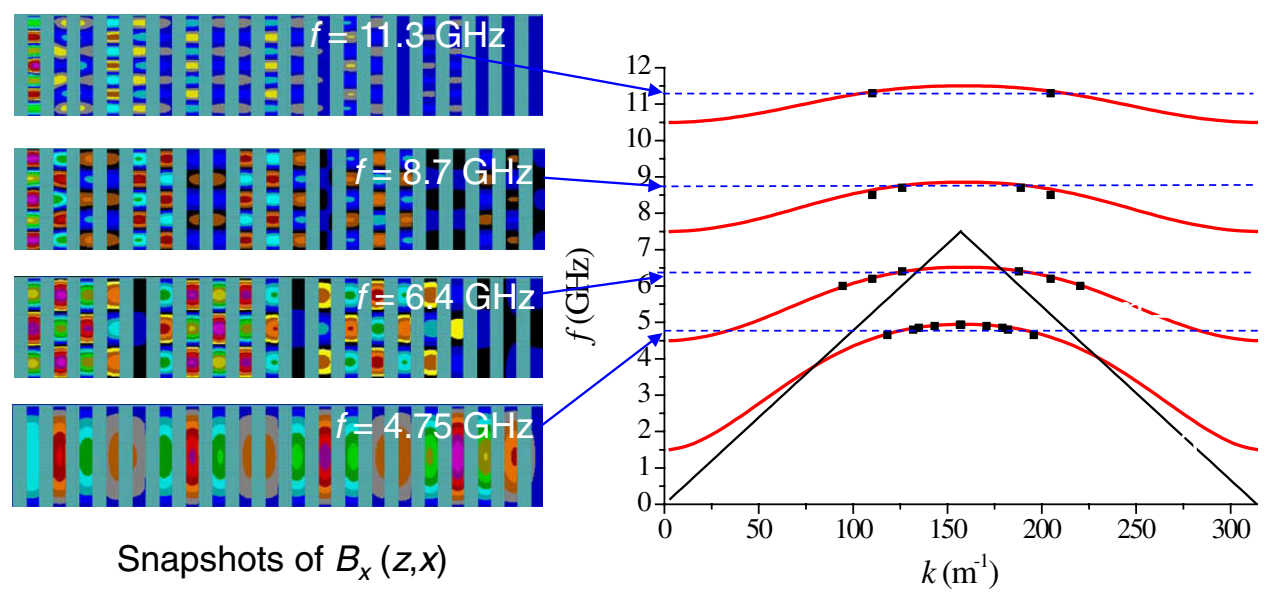

FIG. 8. Simulated contour plots of $B_{x}$ in the $x-z$ plane $(y=-1 \mathrm{~mm})$ at fixed time, generated by a current driver operating at fixed frequencies in each of the four lowest symmetric modes. The grating has sidewalls, where $B_{x}$ is seen to vanish. On the right is shown the theoretical 3D dispersion relation (red). Some results of simulations where the wave numbers $k$ were determined are indicated by squares. 
patterns. This figure shows that frequencies much greater than the $2 \mathrm{D}$ maximum frequency can propagate on a grating with sidewalls.

In Fig. 9(a) we show a simulated contour plot of $B_{x}$ for an antisymmetric mode, with a current driver of frequency $5.3 \mathrm{GHz}$ placed at $x=2.5 \mathrm{~cm}$ in the leftmost groove. The grating has sidewalls. The $y$ coordinate is just below the top of the teeth, so that the teeth appear as field-free zones. According to our theory, the wave numbers for this frequency are 114 and $200 \mathrm{~m}^{-1}$, corresponding to forward and backward waves, respectively. Assuming that the latter is negligible compared to the former (which is not true), we use our model to calculate the field $B_{x}$ as a function of $x, z$, and $t$, with the $y$ coordinate $=0$. Successive grooves are phase shifted by the Floquet factor $e^{i k L}$. By choosing the time appropriately, and adjusting the scale, we generated the theoretical contour plot shown in Fig. 9(b). Since it does not contain the small backward wave, it is not exactly comparable to the simulation, but there is a reasonable similarity.

In Fig. 10 we show the results of 3D simulations performed for our grating without sidewalls, as was the case in the experiment reported in Ref. [11]. Here the current driver placed at $x=0$ was used at several discrete frequencies, and the "range" command of MAGIC was used to obtain the $z$ dependence of $B_{x}$ along the line $x=0, y=$ $2 \mathrm{~mm}$. From the FFT of these, the wave numbers $k$ and $K-k$ were determined, and they are shown in Fig. 10 as circles, triangles, and squares for the three lowest symmetric modes. The theoretical predictions are made using those values of $q$ that reproduce the three symmetric band heads seen in Fig. 7. The corresponding dispersion curves are shown in red, while the 2D prediction is shown in green. The forward and backward light lines are shown in black,

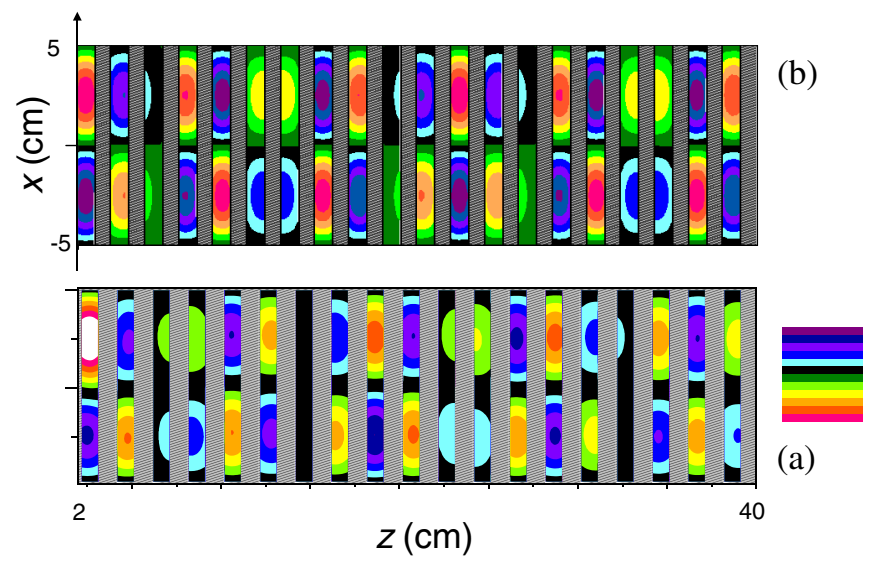

FIG. 9. (a) Simulated contour plot of $B_{x}$ in the $x-z$ plane $(y=$ $-1 \mathrm{~mm}$ ) at fixed time, generated by a current driver operating at $5.3 \mathrm{GHz}$ in the lowest antisymmetric mode. (b) Theoretical contour plot of $B_{x}$ in the $x-z$ plane $(y=-0 \mathrm{~mm})$ at fixed time, corresponding to the forward wave. The time has been chosen to maximize resemblance, and the overall scale has been freely adjusted.

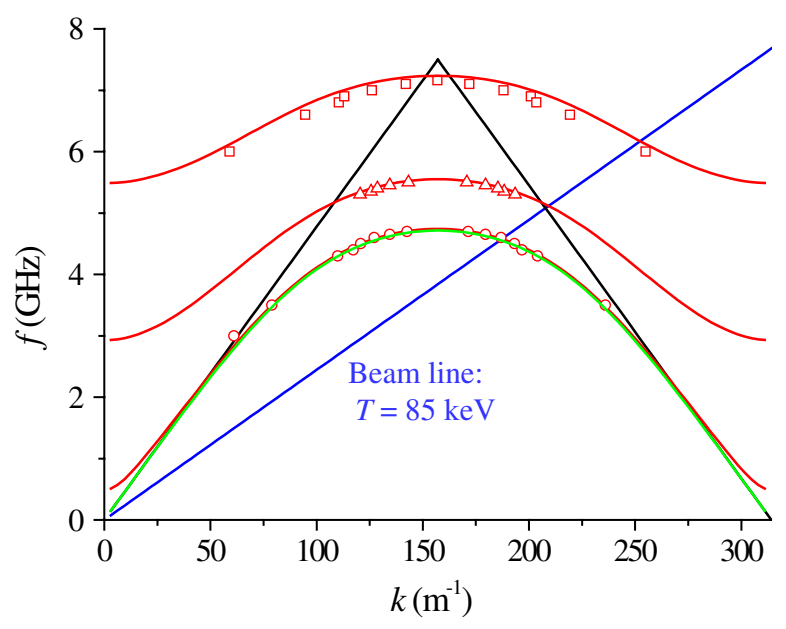

FIG. 10. The three lowest branches of the 3D dispersion relation for the grating without sidewalls, with $q$ chosen to yield the correct band heads, are shown in red. The 2D dispersion relation is shown in green. Black lines represent the forward and backward light lines, while a beam line corresponding to $85 \mathrm{keV}$ is shown in blue. The intersection of the beam line with the two highest branches could generate coherent SP radiation at that frequency. For the lowest branch, the intersection takes place at a sub-SP frequency, and coherent radiation can occur only at harmonics.

and the beam line for a typical energy of $85 \mathrm{keV}$ is shown in blue. The overall agreement between theory and simulation is good, and the validity of the $2 \mathrm{D}$ theory for the lowest mode is obvious. However, the beam line also intersects two higher frequency modes, one just at the backward light line, and the other well beyond it. These intersections correspond to allowed coherent SP radiation, in contrast to the lowest mode. If these higher modes could be excited, it would be possible to have coherent SP radiation without the necessity of bunching on the harmonic of the evanescent wave. We emphasize that this is not the evanescent wave escaping as coherent SP radiation, but simply the usual SP radiation becoming monochromatic and coherent due to the bunching at that frequency.

An important aspect of the excitation of the evanescent wave is the coefficient $\alpha_{p}$ that appears in Eq. (2.5) and which determines the exponential decrease of the field with height above the grating for the Floquet component whose wave number is $k+p K$. In order to excite the evanescent wave with a beam, the beam should pass within a distance of order $1 / \alpha_{0}$ from the top of the grating. According to our theory, this quantity depends on the wave number, but not upon which branch of the dispersion relation one is functioning. The essential relation is

$$
\alpha_{p}=\sqrt{(k+p K)^{2}-\left[\omega_{2 \mathrm{D}}(k)\right]^{2}},
$$

where $\omega_{2 \mathrm{D}}(k)$ denotes the 2D frequency associated with the wave number $k$. This is to be understood as follows: if two surface waves of different frequency $\omega_{3 \mathrm{D}}$ but same 
wave number $k$ are excited, they will have the same value of $\alpha_{0}$. This claim can be tested by exciting waves of fixed frequency with the current driver, and observing the quantity $B_{x}$ as a function of $z$ for $x=0, t$ fixed, and two positive values of $y$. From the FFTs of these curves we find an interval of $k$ in which the best value lies. This interval is swept to find that value of $k$ such that the quantity $\left|\int_{a}^{b} d z B_{x}\left(z, y_{j}\right) e^{i k z}\right|$ attains its maximum. We then use the "Fit" procedure in MATHEMATICA to obtain the complex coefficients $C_{1}$ and $C_{2}$ of the best fit of the form $C_{1} e^{i k z}+$ $C_{2} e^{i(K-k) z}+$ c.c. This is performed for the different values of $y_{j}$, and the corresponding values of $\alpha_{0}$ are obtained from the following expressions:

$$
\begin{gathered}
\alpha_{0}(k)=-\mathfrak{R}\left[\ln \left(\left.C_{1}\right|_{y_{1}} /\left.C_{1}\right|_{y_{2}}\right)\right] /\left(y_{1}-y_{2}\right), \\
\alpha_{0}(K-k)=-\mathfrak{R}\left[\ln \left(\left.C_{2}\right|_{y_{1}} /\left.C_{2}\right|_{y_{2}}\right)\right] /\left(y_{1}-y_{2}\right) .
\end{gathered}
$$

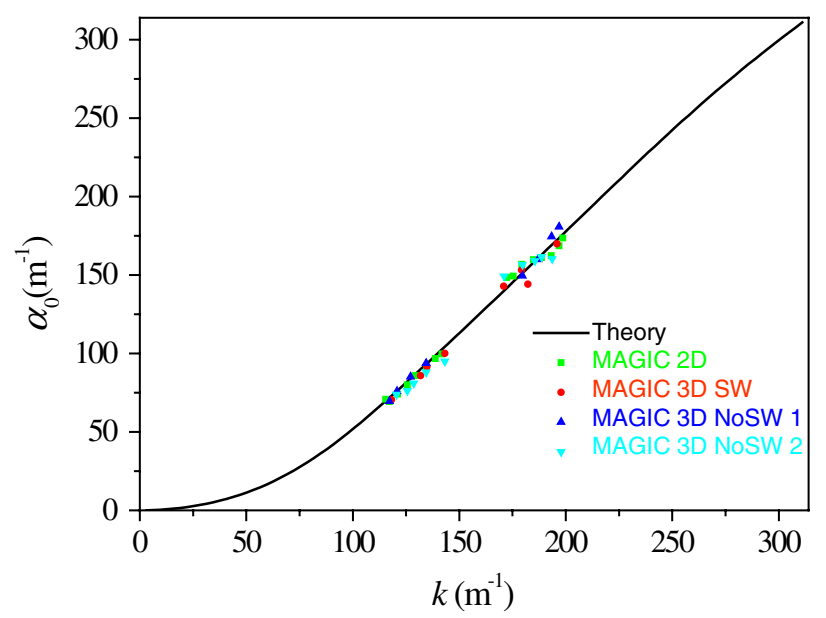

FIG. 11. The coefficient $\alpha_{0}$ [see Eq. (2.5)] for the lowest Floquet component as a function of wave number. According to theory, shown in black, $\alpha_{0}$ depends only on the wave number, and not on the frequency. Simulations in two and three dimensions, with and without sidewalls, were used to generate the points indicated, which tend to lie near the theoretical prediction.
For consistency, the coefficients $C_{i}$ at different heights must have the same phase, and this was typically true, to within 0.1 .

The results of extracting the coefficient $\alpha_{0}$ are displayed in Fig. 11. The theoretical curve is obtained from the 2D dispersion relation, while the simulations involved 2D, 3D with sidewalls, and two different 3D frequency bands with no sidewalls. To a fair degree, these empirically determined values all lie close to the theoretical expectations. In the experiment described in Ref. [11], typical $k$ values of $180 \mathrm{~m}^{-1}$ were found, for which the e-folding height is about $6 \mathrm{~mm}$. In the higher Brillouin zones with $p>0$, much greater values of $\alpha_{p}$ are reached. If one wished to excite such Floquet components with a beam, it would have to pass very close to the grating's surface.

A simple illustration of the predictive power of the theory is provided by the pair of equations (2.24). A 3D simulation of the grating with sidewalls was performed with the current driver operating at $4.7 \mathrm{GHz}$. The field components $B_{y}, B_{z}$, $E_{y}$, and $E_{z}$ were determined as a function of $t$, at $x=w / 2$. At this point the factor $\tan (q x)=1$; the model predicts that the curves of $B_{y}(t)$ and $(q / \omega) E_{z}(t+T / 4)$ should be identical. In Fig. 12(a) these curves are plotted, the former in solid red, the latter in dotted black. The agreement is quite good. Similarly, the prediction that $B_{z}(t)$ and $(q / \omega) E_{y}(t-$ $T / 4$ ) should be identical is tested in Fig. 12(b), with satisfactory if not perfect agreement.

If the grating were equipped with sidewalls, the possibility of exciting some of the higher frequency modes should be considered. To illustrate this, we show in Fig. 13 the dispersion relation for the four lowest modes, extended over two Brillouin zones. The forward and backward light lines are shown, along with the beam line for energy $61 \mathrm{keV}$. Such a beam intersects each branch of the dispersion relation, but only the intersection with the lowest branch occurs in the triangle bounded by the light lines. In this case, the frequency is too low to correspond to allowed SP radiation, and it can only occur on harmonics. However, the other three intersections occur at allowed SP frequencies, and coherent SP radiation at well-defined

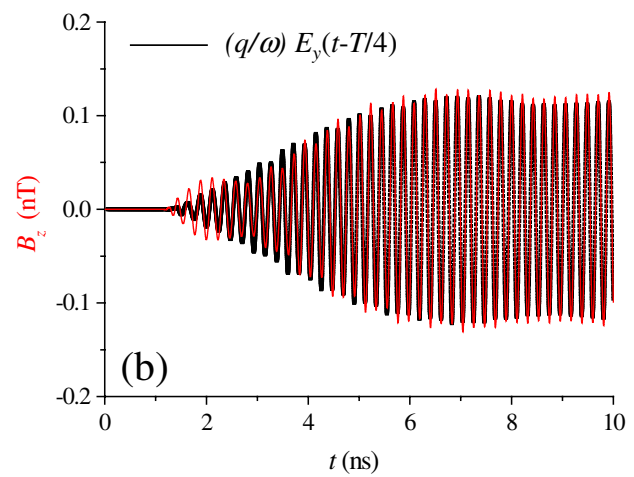

FIG. 12. Tests of the predictions given in Eq. (2.24). The rescaled and time-shifted electric field components (black) are compared with the magnetic field components (red), as determined in a $3 \mathrm{D}$ simulation with sidewalls at $4.7 \mathrm{GHz}$. (a) $B_{y}$ and $E_{z}$; (b) $B_{z}$ and $E_{y}$. 


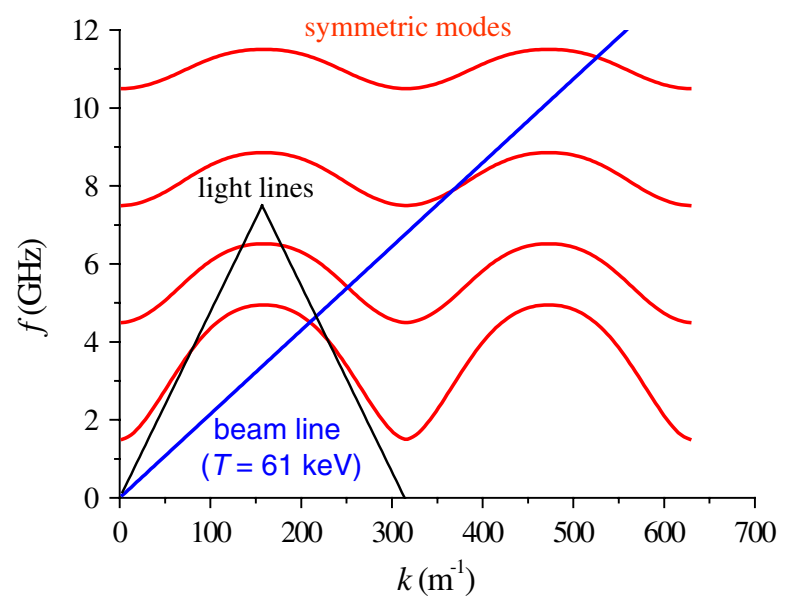

FIG. 13. Dispersion relation for the four lowest symmetric modes of the grating with sidewalls, extended over the first and second Brillouin zones (red). The forward and backward light lines are shown (black), along with a beam line corresponding to $61 \mathrm{keV}$ (blue). The intersections with the high frequency modes occur at frequencies which correspond to allowed SP radiation.

angles could be produced, provided these evanescent waves could be excited. It is not the evanescent wave itself which is radiated, but rather the coherent SP radiation due to the bunching at that frequency.

\section{Comparison with results of McVey and collaborators}

As stated in the Introduction, the problem of a grating enclosed by both sidewalls and a roof was treated by McVey and collaborators many years ago. They considered a section of rectangular waveguide whose lower plate formed a rectangular grating. It is straightforward to adapt our model to theirs. All that is necessary is to replace the factor $e^{-\alpha_{p} y}$ appearing in Eq. (2.5) by $\cosh \left[\alpha_{p}(b-y)\right] /$ $\cosh \left(\alpha_{p} b\right)$, where $b$ denotes the height of the roof measured from the top of the grating. This ensures that the tangential electric field vanishes on the roof. Everything goes through in our derivation of the matrix $\bar{R}$, except that the quantity $\alpha_{p}$ must be replaced by $\alpha_{p} \tanh \left(\alpha_{p} b\right)$ wherever it appears. This change, which is minor if $\alpha_{p} b \gg 1$, is very important, since it allows $\alpha_{p}$ to take on imaginary values while keeping the eigenvalues of the matrix $\bar{R}$ real. This means that solutions to Eq. (2.16) exist for imaginary $\alpha_{p}$. This does not happen in the AB approach, since the determinant becomes complex for imaginary $\alpha_{p}$.

To illustrate the relation between our approach and that of Ref. [23], we use their parameters (in inches), period $L=0.07$, groove depth $H=0.260$, groove width $A=$ 0.035 , grating width $w=0.622$, and height $b=0.311$. In Fig. 14(a) we show in blue the $2 \mathrm{D} \mathrm{AB}$ calculation for this grating if its width were infinite and without roof. If the grating width is kept infinite, but the roof is taken into account as shown above, the red dotted curves are obtained. The existence of several branches is characteristic of gratings where the groove depth exceeds the period. In the absence of a roof these branches are confined to the zone bounded by the light lines. When the roof is included, these curves connect smoothly to modes that at long wavelength become waveguide modes.

In Fig. 14(b) are shown the results of our 3D theory with sidewalls (but no roof) in blue, and its extension to a roof as red dotted lines. Comparison of the latter with the three branches shown in Fig. 3 of Ref. [23] shows excellent agreement. It should be noted that in the region bounded by the light lines the roofless and roofed gratings have almost identical properties. In fact, for the lowest frequency, the agreement is nearly perfect everywhere.

\section{CONCLUSION}

In this paper we have presented numerous arguments in support of our theory for the 3D grating. It is based on a simple extension of the $2 \mathrm{D} \mathrm{AB}$ theory for a lamellar grating, as given by Eq. (1.2). We have offered evidence in its favor based on 3D simulations and experimental measurements of the transmission coefficients. A comparison with
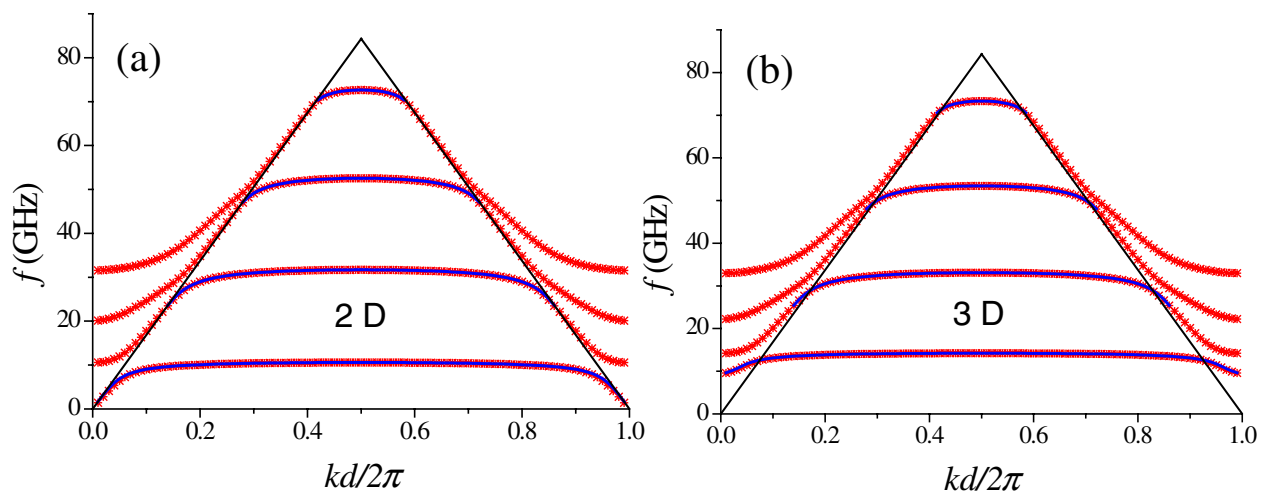

FIG. 14. Comparison of our theory with that of McVey et al., Ref. [23], for the grating discussed therein. (a) Blue curves, 2D Andrews and Brau dispersion relation. Red dotted curves, our extension of that to a roofed grating. (b) Blue curves, our 3D dispersion relation. Red dotted curves, the extension of our theory to a roofed grating. The latter agree with results shown in Ref. [23]. 
earlier established work on an enclosed grating lends support to our theory. We have also found that even a grating with no sidewalls exhibits a frequency band structure similar to that of a grating with sidewalls. What remains to be shown is the relevance to future experimental work, which might establish some of the features we showed in Figs. 10 and 13.

Although all of our simulations and measurements concerned our grating, which functions in the microwave frequency domain, there is no intrinsic scale in our theory, and it should be valid at all frequencies. In order to obtain terahertz frequencies with SP free-electron lasers, gratings of much smaller periods must be used. We think that our theory, together with simulations using PIC codes such as MAGIC, can provide guidance in designing such gratings.

\section{ACKNOWLEDGMENTS}

We acknowledge many useful discussions with Charles Brau, and we thank him for bringing Ref. [23] to our attention.

[1] S. J. Smith and E. M. Purcell, Phys. Rev. 92, 1069 (1953).

[2] H. L. Andrews and C.A. Brau, Phys. Rev. ST Accel. Beams 7, 070701 (2004).

[3] H. L. Andrews, C.H. Boulware, C.A. Brau, and J.D. Jarvis, Phys. Rev. ST Accel. Beams 8, 050703 (2005); 8, 110702 (2005).

[4] J. T. Donohue and J. Gardelle, Phys. Rev. ST Accel. Beams 8, 060702 (2005).

[5] D. Li, Z. Yang, K. Imasaki, and Gun-sik Park, Phys. Rev. ST Accel. Beams 9, 040701 (2006).

[6] B. Goplen, L. Ludeking, D. Smithe, and G. Warren, Comput. Phys. Commun. 87, 54 (1995).

[7] Vinit Kumar and Kwang-Je Kim, Phys. Rev. E 73, 026501 (2006).
[8] B. K. Skrynnik, V. K. Korneyenkov, and M. Yu. Demchenko, Telecommun. Radio Eng. 55, 170 (2001).

[9] R.E. Collin, Foundations for Microwave Engineering (McGraw-Hill, New York, 1992), 2nd ed., Chap. 8.

[10] H. L. Andrews, C. A. Brau, J. D. Jarvis, C. F. Guertin, A. O'Donnell, B. Durant, T. H. Lowell, and M. R. Mross, Phys. Rev. ST Accel. Beams 12, 080703 (2009).

[11] J. Gardelle, L. Courtois, P. Modin, and J. T. Donohue, Phys. Rev. ST Accel. Beams 12, 110701 (2009).

[12] J. Gardelle and J.T. Donohue, IEEE Trans. Electron Devices 56, 769 (2009).

[13] Amit S. Kesar, Phys. Rev. ST Accel. Beams 13, 022804 (2010).

[14] Kwang-Je Kim and Vinit Kumar, Phys. Rev. ST Accel. Beams 10, 080702 (2007).

[15] J. Urata, M. Goldstein, M. F. Kimmit, A. Naumov, C. Platt, and J.E. Walsh, Phys. Rev. Lett. 80, 516 (1998).

[16] D. Li, K. Imasaki, Z. Yang, G-S. Park, S. Miyamoto, S. Amano, and T. Mochizuki, Jpn. J. Appl. Phys. 46, 601 (2007).

[17] D. Li, K. Imasaki, X. Gao, Z. Yang, and Gun-Sik Park, Appl. Phys. Lett. 91, 221506 (2007).

[18] D. L. Li, K. Imasaki, X. Gao, J. Hou, and Z. Yang, Terahertz Sci. Technol. 1, 221 (2008).

[19] H. L. Andrews, J. D. Jarvis, and C. A. Brau, J. Appl. Phys. 105, 024904 (2009).

[20] J. D. Jarvis, H. L. Andrews, and C. A. Brau, Phys. Rev. ST Accel. Beams 13, 020701 (2010).

[21] F. S. Rusin and G. D. Bogomolov, Proc. IEEE 57, 720 (1969).

[22] K. Mizuno, S. Ono, and T. Shibata, IEEE Trans. Electron Devices 20, 749 (1973).

[23] B. D. McVey, M. A. Basten, J. H. Booske, J. Joe, and J. E. Scharer, IEEE Trans. Microwave Theory Tech. 42, 995 (1994).

[24] A. Sommerfeld, Electrodynamics (Academic Press, New York, 1952).

[25] Wolfram Research, Inc., MATHEMATICA, Version 6.0, Champaign IL, 2007.

[26] J.W.S. Rayleigh, Theory of Sound, Vol. II (Dover Publications, New York, 1945), 2nd ed. 